\title{
Raw-to-raw: Mapping between image sensor color responses
}

\author{
Rang Nguyen Dilip K. Prasad Michael S. Brown \\ School of Computing, National University of Singapore \\ nguyenho@comp.nus.edu.sg | dilipprasadegmail.com | brownecomp.nus.edu.sg
}

\begin{abstract}
Camera images saved in raw format are being adopted in computer vision tasks since raw values represent minimally processed sensor responses. Camera manufacturers, however, have yet to adopt a standard for raw images and current raw-rgb values are device specific due to different sensors spectral sensitivities. This results in significantly different raw images for the same scene captured with different cameras. This paper focuses on estimating a mapping that can convert a raw image of an arbitrary scene and illumination from one camera's raw space to another. To this end, we examine various mapping strategies including linear and non-linear transformations applied both in a global and illumination-specific manner. We show that illumination-specific mappings give the best result, however, at the expense of requiring a large number of transformations. To address this issue, we introduce an illumination-independent mapping approach that uses white-balancing to assist in reducing the number of required transformations. We show that this approach achieves state-of-the-art results on a range of consumer cameras and images of arbitrary scenes and illuminations.
\end{abstract}

\section{Introduction}

Camera manufactures apply photo-finishing operations (e.g. tone-mapping, white-balancing, etc.) before saving images in the standard rgb format (i.e. JPEG). For many computer vision tasks, such in-camera processing must be undone to map sRGB values back to physically meaningful values (e.g. see [5, 8, 9, 15, 21]). Most consumer cameras now allow images to be saved in raw format that represents a minimally processed image obtained from the camera's sensor. This format is desirable for computer vision tasks as the raw-rgb values are known to be linearly related to scene radiance [5, 15], thereby avoiding the need to undo photofinishing. One drawback, however, is that manufacturers have yet to agree on a standard raw format. As a result, the raw-rgb values are device specific and raw images of the same scene and illumination from different cameras can differ significantly (see Fig. 1,

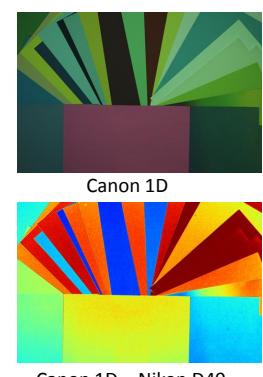

Canon 1D - Nikon D40
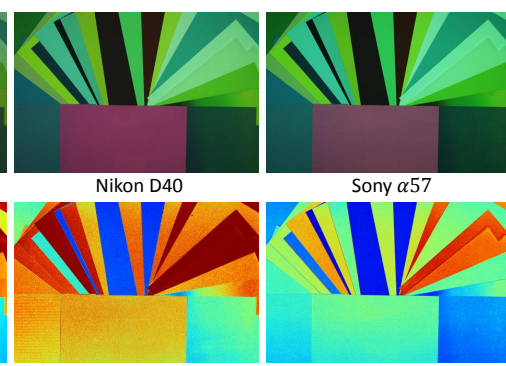

Canon 1D - Sony $\alpha 57$

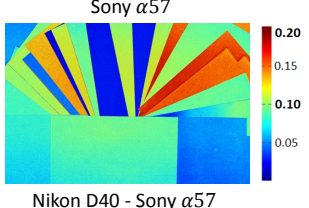

Nikon D40 - Sony $\alpha 57$
Figure 1. Top row shows three raw images taken from Canon 1D, Nikon D40, and Sony $\alpha 57$. Bottom row shows the numerical difference between the raw images.

The goal of this paper is to find a mapping between different cameras' raw-rgb colorspaces. This is useful for a variety of reasons from comparing scene objects between different cameras to mosaicing raw images from multiple cameras. This problem is inherently challenging since the raw-rgb values represent a projection of scene radiance onto only three color channels, where the projection differs due to the unique spectral responses of sensors found in different camera makes and models.

Contribution The contribution of our work is to conduct an analysis of a range of strategies to perform the raw-to-raw mapping between cameras. In particular, linear transformations, radial basis functions, gaussian process regression, and polynomial fitting are evaluated in a global mapping manner (all illuminations combined) and an illuminationspecific manner. From this analysis, a new calibration and mapping method is proposed that uses two linear transformations together with a white-balancing step to provide a compact solution that offers state-of-the-art results. The effectiveness of this approach is demonstrated on a wide range of cameras and images. In addition, a data set for studying the raw-to-raw mapping problem has been assembled with over 250 raw images from eight different cameras of color charts and various scenes under different illuminations for calibration and testing. 


\section{Related work and preliminaries}

There is not a great deal of prior works addressing the mapping between raw colorspaces. Most work focuses on the related problem of making cameras colorimetric by finding a mapping between a camera's raw-rgb values and a color chart with known CIE XYZ values. This is mainly done by a simple $3 \times 3$ linear transform (e.g. [17]). Work by Hong et al. [13] examined the use of a polynomial function but for a single illumination only. Other related works attempt to recover the full spectral response of the cameras (e.g. [14, 20, 18]) through often complex procedures that may not be practical for mainstream use. Moreover, knowing the spectral responses does not directly reveal the best approach to use to map between different cameras.

Adobe has arguably been the most serious in addressing this issue. Adobe proposed a standard raw space termed Digital Negative (DNG) in 2004, however, few manufacturers have adopted it. Adobe provides an SDK ${ }^{1}$ that can convert a wide range of camera models into Adobe's DNG format. Adobe has also provided a report describing the conversion [1], which is explained in more detail in Sec.5.

Given the lack of prior work, we begin our preliminaries by discussing the problem of color constancy/whitebalancing given its relevance to the raw-to-raw mapping problem and its use in our proposed method described in Sec. 4.

Preliminaries We start with the image formation model in the spectral domain, where an $\operatorname{rgb}$ image $I$ can be written as:

$$
I(\mathbf{x})=\int_{\omega} R_{c}(\lambda) S(\mathbf{x}, \lambda) L(\lambda) d \lambda,
$$

where $\lambda$ represents the wavelength, $\omega$ is the visible spectrum $380-720 \mathrm{~nm}, R_{c}$ is the camera's spectral response, and $c$ is the color channel $c=r, g, b$. The term $S(\mathbf{x}, \lambda)$ represents the scene's spectral response at pixel $\mathbf{x}$ and $L(\lambda)$ is the lighting in the scene, assumed to be spatially uniform.

The color constancy problem can be expressed as follows (dropping the spatial location $\mathbf{x}$ for simplicity):

$$
\begin{aligned}
& I^{L_{1}}=\int_{\omega} R_{c}(\lambda) S(\lambda) L_{1}(\lambda) d \lambda \\
& I^{L_{2}}=\int_{\omega} R_{c}(\lambda) S(\lambda) L_{2}(\lambda) d \lambda,
\end{aligned}
$$

where $R_{c}$ and $S$ are assumed to be fixed (i.e. images $I^{L_{1}}$ and $I^{L_{2}}$ are taken by the same camera). The difference between the images is due to the different lightings $L_{1}$ and $L_{2}$. The goal in color constancy is to compute a transformation to make the image values of these two images as similar as possible. It is generally accepted that a diagonal $3 \times 3$ matrix $T$ is sufficient to map between these images [4, 7, 12], where the diagonal matrix $T$ maps the rgb values of $I^{L_{1}}$ to $I^{L_{2}}$. When the target illumination is not specified (as is

1 http://www.adobe.com/support/downloads / product $\cdot$ jsp?product $=106$ \&plat form=Windows often the case), the problem becomes one of mapping the source illumination, $L_{1}$, to a canonical illumination. This is typically referred to as white-balancing where an observed or estimated white-point (denoted as $r_{w}, g_{w}, b_{w}$ ) in the image $I^{L_{1}}$ maps to the rgb values $(1,1,1)$. This means that the diagonal entries of $T$ are $1 / r_{w}, 1 / g_{w}, 1 / b_{w}$. Whitebalancing is a thoroughly studied topic and a variety of techniques to estimate $T$ exist (e.g. [2, 10, 11, 6], for an excellent survey see [12]).

The raw-to-raw mapping problem can be similarly expressed as:

$$
\begin{aligned}
& I_{1}=\int_{\omega} R_{1 c}(\lambda) S(\lambda) L(\lambda) d \lambda \\
& I_{2}=\int_{\omega} R_{2 c}(\lambda) S(\lambda) L(\lambda) d \lambda,
\end{aligned}
$$

where, in this case, the changing variables are the camera response functions, $R_{1 c}$ and $R_{2 c}$, i.e. images $I_{1}$ and $I_{2}$ are from different cameras. Similar to color constancy, the goal is to compute a mapping, denoted as $f$, such that we can map image $I_{1}$ to appear as image $I_{2}$, i.e.:

$$
I_{2}=f\left(I_{1}\right)
$$

Ideally, this mapping should work for any scene and lighting condition. Since $f$ does not depend on the image's spatial content, it can be considered a colorspace conversion from $I_{1}$ 's raw-rgb to $I_{2}$ 's raw-rgb. While similar to color constancy, the raw-to-raw mapping has to compensate for three different color response functions versus one change in spectral lighting. In addition, this mapping needs to be computed in the face of camera-based metamerism. More discussion about metamerism related to raw-to-raw mapping is included in supplementary material.

To estimate $f$, a number of corresponding raw-rgb samples between the two cameras colorspace is needed. The most direct way to obtain this is by having both cameras image a calibration color chart under several different illuminations as shown in Figure 2 The use of the chart establishes corresponding raw-rgb between cameras (i.e. patches are unique colors); the multiple illuminations help to span the camera's gamut and serve to reveal how scene colors $S$ shift due to illumination change $L$. The question now is how to best estimate $f$ ? We explore this in the next section examining five mapping approaches applied in both a global manner for any type of scene and illumination (denoted as $f^{G}$ ), as well as the more restrictive case of an illuminationspecific transformation, denoted as $f^{L}$, where $L$ is a known illumination.

\section{Evaluating mapping approaches}

\subsection{Mapping methods}

We examine five different transformation approaches for estimating $f$. These are as follows: 


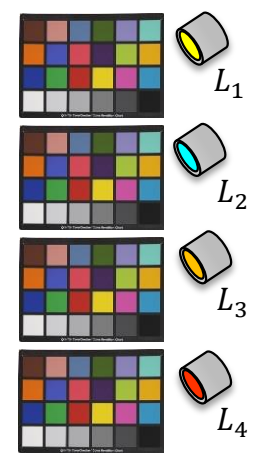

Color chart imaged under different illuminations $L_{i}$

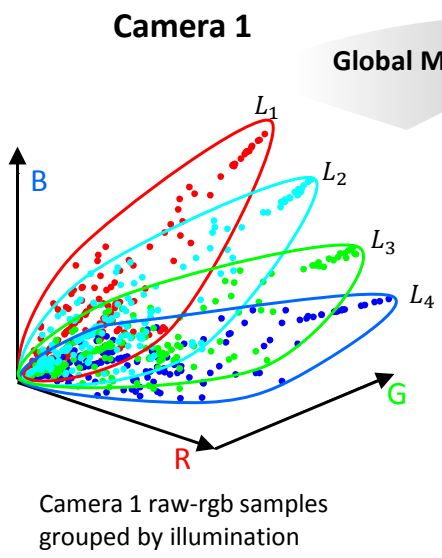

Camera 1

grouped by illumination
Camera 2

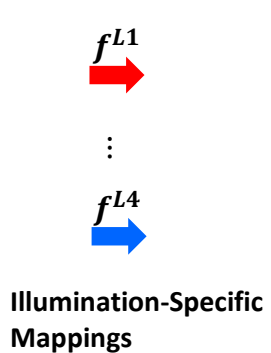

Mappings

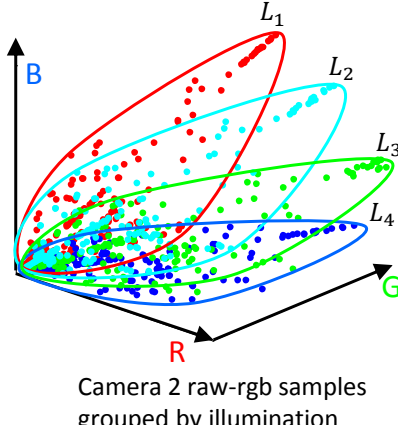

grouped by illumination
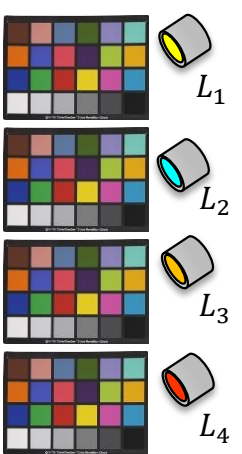

Color chart imaged under different illuminations $L_{i}$

Figure 2. This figure shows the raw-to-raw calibration setup. Images of color calibration charts are taken under several different lighting conditions by the source and target cameras. Mapping between these two cameras' raw-rgb colorspaces can be estimated using a global mapping (all illuminations combined) or via multiple illuminant-specific mappings.

Linear transform $\left(\mathrm{T}_{3 \times 3}\right)$ This uses a simple $3 \times 3$ matrix to perform the mapping. The values can be solved using any standard least-squares method. Unlike color constancy, the transform is a full matrix, not a diagonal.

Linear transform with offset $\left(\mathrm{T}_{3 \times 4}\right)$ This method extends the $3 \times 3$ matrix by adding an $3 \times 1$ offset vector to make an affine transform. The matrix and offsets can be solved using a standard least-squares method.

Polynomial model (Poly) This method was proposed in [13] and extends the linear transformation by adding high-order terms (e.g., $r g, r b, g b, r^{2}, g^{2}, b^{2}$ ). The parameters can be obtained by solving a $3 \times 11$ matrix in a leastsquares manner.

Radial basis functions (RBF) RBF is a well known scatter point interpolation method which is described as: $f(x)=$ $\sum_{i=1}^{N} w_{i} \phi\left(\left\|x-c_{i}\right\|\right)$, where the approximating function $f(x)$ is a linear combination of $N$ radial basis functions $\phi(r)$. Each basic function is controlled by a different center $c_{i}$ estimated from a set of corresponding points given between the source and target space and weighted by an appropriate coefficient $w_{i}$ that can be computed by linear least-squares manner. For more information see [3].

Gaussian process regression (GPR) GPR uses a joint Gaussian distribution for estimation: $f(x) \sim$ $N\left(m, K\left(\theta, x, x^{\prime}\right)\right)$, where $m$ is the mean function value, $K\left(\theta, x, x^{\prime}\right)$ is the covariance matrix between all possible pairs $\left(x, x^{\prime}\right)$ for a given set of hyperparameters $\theta$. For more information see [19].

\subsection{Global versus illumination-specific}

As mentioned in Section 2, we evaluate the different mappings in a global and illumination-specific manner. For the global approach, we estimate the mapping using the five models previously described using all of the colorchart samples under different illuminations. For the illumination- specific method, we use only the color samples for a particular illumination.

The results of these five strategies applied as a global mapping and illumination-specific mapping are shown in Table 1 in columns one and two. To compute the mapping, two Macbeth color charts (24 patches and 140 patches) is imaged under four different lighting conditions: Fluorescent (FL), Incandescent (IN), Halogen (HA), and LED (LE). The source and target cameras shown here are for a Canon 1D and Nikon D4Q 2 For the RBF model, we used one more dataset (paper and paint chips described in Section 5.1 for cross-validation to control the number of central points to avoid overfitting. The residual errors shown in Table 1 were computed on the color calibration charts and are reported as root mean square error (RMSE).

\subsection{Discussion}

It is clear from Table 1 that among all the approaches for modeling $f$, the illumination-specific transformations give the lowest errors. Not surprisingly, the more complex transformation methods such as $\mathbf{R B F}$ and $\mathbf{G P R}$ provide better fitting in both the global and illumination-specific approaches. However, this finding comes at a cost in terms of evaluation time, where RBF and GPR can take several minutes to convert an image, while the other transformations can be done in a matter of seconds.

The obvious downside of the illumination-specific strategy is the need to store several mappings for the most common illuminations. This also requires that when an image is converted its illumination must be known to select the correct transformation. This makes illumination-specific mappings less attractive for practical applications for raw-to-raw mapping.

\footnotetext{
${ }^{2}$ Results for other camera pairs show a similar trend and are reported in the supplemental materials.
} 


\begin{tabular}{|c|c|c|c|c|c|c|c|c|c|c|c|c|c|c|c|}
\hline \multirow{2}{*}{ Light } & \multicolumn{5}{|c|}{ Global (in $10^{-2}$ ) } & \multicolumn{5}{|c|}{ Illumination-specific (in $10^{-2}$ ) } & \multicolumn{5}{|c|}{ Our method (in $10^{-2}$ ) } \\
\hline & $T_{3 \times 3}$ & $T_{3 \times 4}$ & Poly & RBF & GPR & $T_{3 \times 3}$ & $T_{3 \times 4}$ & Poly & RBF & GPR & $T_{3 \times 3}$ & $T_{3 \times 4}$ & Poly & RBF & GPR \\
\hline FL & 1.65 & 1.61 & 1.56 & 1.57 & 1.53 & 1.29 & 1.25 & 1.118 & 1.18 & 1.24 & 1.35 & 1.28 & 1.26 & 1.27 & 1.28 \\
\hline IN & 1.23 & 1.05 & 0.84 & 0.84 & 0.67 & 0.95 & 0.68 & 0.62 & 0.62 & 0.63 & 0.99 & 0.75 & 0.73 & 0.73 & 0.66 \\
\hline HA & 0.77 & 0.64 & 0.38 & 0.38 & 0.35 & 0.52 & 0.32 & 0.29 & 0.29 & 0.30 & 0.55 & 0.37 & 0.37 & 0.37 & 0.32 \\
\hline LE & 0.63 & 0.66 & 0.36 & 0.37 & 0.47 & 0.32 & 0.27 & 0.26 & 0.26 & 0.27 & 0.36 & $\mathbf{0 . 3 3}$ & 0.33 & 0.33 & 0.32 \\
\hline AVG & 1.07 & 0.99 & 0.79 & 0.79 & 0.76 & 0.77 & 0.63 & 0.59 & 0.59 & 0.61 & 0.81 & 0.68 & 0.67 & 0.68 & $\overline{0.65}$ \\
\hline
\end{tabular}

Table 1. The table shows the comparisons of error between all linear and non-linear models in three categories: global, specific and whitebalancing. We used color calibration charts taken under four lighting conditions: Fluorescent (FL), Incandescent (IN), Halogen (HA), and LED (LE). AVG means the average error for all the lightings.

\begin{tabular}{|c|c|c|c|c|c|c|}
\hline & \multicolumn{2}{|c|}{ Canon1D } & \multicolumn{2}{c|}{ Nikon D40 } & \multicolumn{2}{c|}{ Sony $\alpha 57$} \\
\cline { 2 - 7 } & W & C & W & C & W & C \\
\hline Canon 1D & - & - & 3.76 & 5.37 & 4.71 & 5.61 \\
\hline Nikon D40 & 3.65 & 5.27 & - & - & 5.34 & 6.31 \\
\hline Sony $\alpha 57$ & 4.95 & 5.83 & 5.46 & 6.60 & - & - \\
\hline
\end{tabular}

Table 2. The table shows the comparisons of percentage error (in $\%)$ between white points $(\mathbf{W})$ and color points $(\mathbf{C})$ by the global transform.

It is important to note that the fitting errors from the global methods were not distributed equally for all color values. For example, raw-rgb values that represent illumination (i.e. the gray/white patches on the colorchart) report a lower error than the average error of the other colors. Table 2 shows these errors using the $T_{3} \times 4$ transformation in terms of percentage error to remove the effect of the magnitude of the raw-rgb values. This finding led us to develop an alternative to the illumination-specific method that provided similar results but in a much more compact manner. This approach is described in the following section.

\section{Proposed illumination-independent method}

Figure 3-(A) overviews our proposed calibration procedure, and Figure 3-(B) describes how to perform the raw-to-raw conversion. Both are described in detail below.

Calibration The approach starts by computing a global transformation $f^{G}$ between the two cameras raw-rgb colorspaces. Next, color values for each illumination are transformed by a white-balance transformation which uses the white-patches on the color chart to estimate the white points. This results in both cameras illuminations being mapped to a canonical illumination. Finally, a mapping, $f^{L_{c}}$, is computed between these transformed colorspaces, where the superscript $L_{c}$ denotes the canonical illumination. This means that our method needs to compute only two mappings, a global mapping, $f^{G}$, and a canonicalized mapping $f^{L_{c}}$.

Raw-to-raw mapping Consider an input source image $I_{s}^{L}$ taken under an unknown illuminant $L$. Our goal is to transform this to another camera's colorspace as if it was taken of the same scene and under the same illumination, i.e. to target image $I_{t}^{L}$. First, the white-point of $I_{s}^{L}$ is estimated, i.e. $\left(r_{w s}, g_{w s}, b_{w s}\right)$. This can be done with any robust white-balancing algorithm (e.g. [4, 7, 12]). Next the source image is white-balanced using the estimated $T_{w s}$. This results in a new image, denoted as $I_{s}^{L_{c}}$, that has a canonical illumination. From this, the image can be mapped to the canonical lighting in target raw-rgb space via $I_{t}^{L_{c}}=f^{L_{c}}\left(I_{s}^{L_{c}}\right)$.

After conversion using $f^{L_{c}}$ it is still necessary to map the target image $I_{t}^{L_{c}}$ back to the correct input illumination. We cannot use the estimated white-point $\left(r_{w s}, g_{w s}, b_{w s}\right)$, since this was estimated in the source colorspace. Instead, we find the target white-point by using the global transform, such that $\left(r_{w t}, g_{w t}, b_{w t}\right)=f^{G}\left(r_{w s}, g_{w s}, b_{w s}\right)$. As previously discussed, while the global transformation $f^{G}$ is not ideal for all colors, it has acceptable performance on color values associated with illuminations. Once we have this target white point, we can obtain the desired $I_{t}^{L}$ by applying the inverse white-balance transform, $T_{w t}^{-1}$.

Transformation used Table 1 (last column) shows our proposed method implemented using the five different transformation methods discussed in Section 3.1. For the mapping models, while the non-linear mapping models perform better, the linear offset model follows them closely. Further, the inherent simplicity and lower dimensionality of this model scores over the non-linear models. Thus, we choose to implement our canonical illumination method using linear offset model (i.e. $T_{3 \times 4}$ ) for estimating $f^{G}$ and $f^{L_{c}}$ used in our method.

\section{Experiments and results}

We compare our method with the method presented in [13] and the Adobe's Digital Negative (DNG) software. The experiments are tested on two different data sets. The first is an image set of paint and paper samples for which explicit point correspondences can be extracted easily (denoted as the controlled image set). The second data set is a series of 

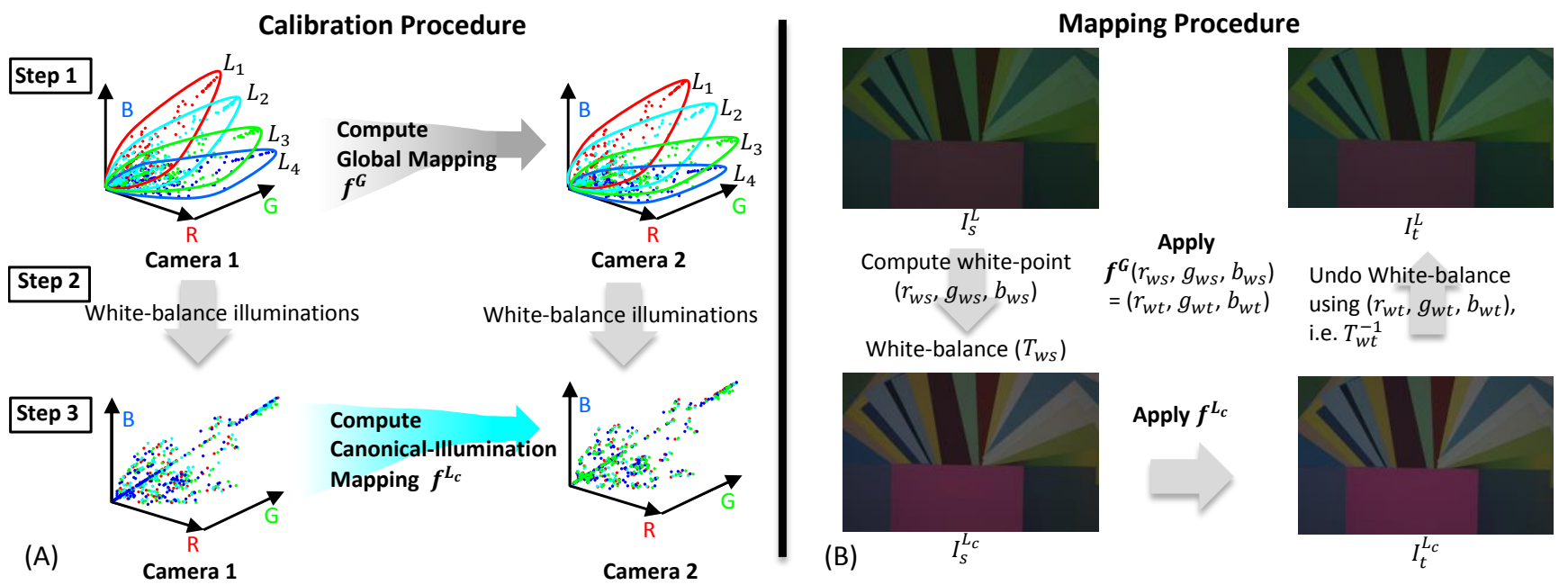

Figure 3. This figure shows the overview of our raw-to-raw calibration and conversion approach. (A) shows the steps of our calibration procedure. A global mapping $f^{G}$ is computed using all of the color chart points. White-balancing is then applied to the color charts values from both cameras. Next a mapping on the canonical-illumination, $f^{L_{c}}$ is computed. (B) illustrates the conversion procedure (see paper for details).

outdoor scenes (termed outdoor image set) taken by multiple cameras from the same location. The global and canonical illuminations mapping are computed only once as described in Section 4 from raw-rgb samples obtained from two Macbeth color charts imaged under six illuminations with four lighting conditions (two types of fluorescent, two types of incandescent, halogen, and LED light). Calibration and conversion code is written in Matlab and takes less than a second for raw-to-raw conversion. Matlab source code and data sets are available on the project webpage ${ }^{3}$

Adobe outlines their procedure for DNG conversion in [1] and recommends estimating a $T_{3 \times 3}$ mapping for only two different lighting conditions, $L_{1}$ and $L_{2}$. It is recommended that these lighting conditions be "far apart" from one another in terms of color temperature. From this, two transformations can be computed, $T^{L_{1}}$ and $T^{L_{2}}$. Given a raw input image the illumination is obtained from the raw file's metadata (i.e. the white-balance setting), and the raw-to-raw transformation, $f$, is estimated as: $f=$ $w_{1} T^{L_{1}}+w_{2} \mathbf{T}^{L_{2}}$ where the weights $w_{1}$ and $w_{2}$ are based on the similarity of the input image's illuminant, $L$ to the two illuminates $L_{1}$ and $L_{2}$, s.t. $w_{1}+w_{2}=1$. Details to how these weights are computed is not explicitly given.

The Adobe SDK does not explicitly map between two different cameras, but instead maps images to the DNG format which acts as canonical color space. Note that this is different from our proposed canonical illumination space. To make sure that the reported errors are in the same scale space, we use DNG space to compute a direct camera to camera mapping. This is done by converting the two im-

$\sqrt[3]{\text { www.comp.nus.edu.sg/ whitebal/raw_2_raw/index. }}$ html ages from the two cameras, denoted as $I_{s}$ and $I_{t}$, to their DNG representation $I_{s}^{D}$ and $I_{t}^{D}$ using the DNG software. We can now compute a $3 \times 3$ linear transformation $T_{s}^{D}$ that maps $I_{s}$ to $I_{s}^{D}$. The transform $T_{t}^{D}$ can be computed similarly for $I_{t}$. Since $I_{s}^{D}$ and $I_{t}^{D}$ are in canonical space, we have $I_{t}=\left(T_{t}^{D}\right)^{-1} \times T_{s}^{D} \times I_{s}$. We found that the residual errors in computing these $T_{s}^{D}$ and $T_{t}^{D}$ were very low, indicating that this was a reasonable strategy for comparison.

While the images in our data set have been taken with the same exposure settings, small differences attributed to lens optics and sensor gain require the use of a scale factor $k$ to adjust the two raw images to have similar exposure before evaluating the residual error. Here $k=\sum_{\mathbf{x}} I_{t, \mathbf{x}}^{l} / \sum_{\mathbf{x}} \hat{I}_{t, \mathbf{x}}^{l}$, where $\mathbf{x}$ is the pixel location, $I_{t}$ is the ground-truth target image, and $\hat{I}_{t}$ is a converted raw-image.

\subsection{Controlled image set}

This data set consists of images of colored paint chips and paper samples. Examples are shown in Figure 4 These are imaged under indoor fluorescent, tungsten and outdoor daylight illuminations with the following cameras: Canon 1D Mark III, Canon EOS 600D, Nikon D40, Nikon D5200, Olympus E-PL6, Panasonic DMC-GX1X, Samsung NX2000, and Sony $\alpha 57$. Images are aligned using a homography and further cropped to ensure that only the colors are being compared. A jet map is used to show the pixel error between the transformed raw-rgb images and the ground truth raw-rgb images.

We also show the following pixel error statistics: mean $(\mathrm{M})$ error, $25 \%$ quartile $(Q 1)$ error, $50 \%$ quartile $(Q 2)$ error (i.e. median error) and $75 \%$ quartile (Q3) error. Examples of raw-to-raw conversions are shown in Figures 5 and 6 

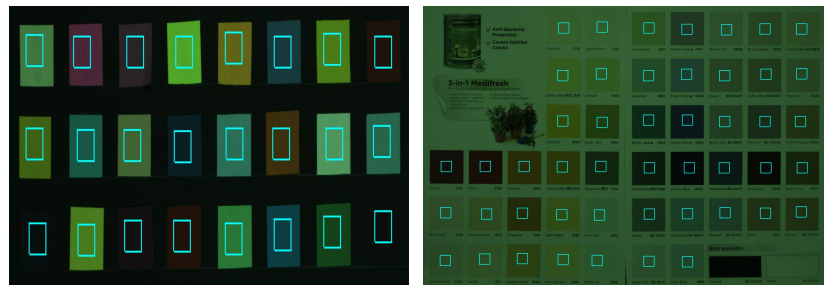

Figure 4. Example images of the controlled image set of paint chips and paper samples. The cyan rectangles are regions used for extracting the raw values.

The error map as well as the mean and quartile errors show that the proposed method results in lower conversion errors. See supplemental material for additional results.

\subsection{Outdoor image set}

Figure 7 shows examples from the outdoor image data set. Care is taken to align and crop the images to be similar, however, there are still some slight misalignment. This makes it hard to evaluate the error by using point wise subtraction. Instead, we examine how well aligned the raw-rgb color histograms of the ground truth and converted image are. The symmetric Kullback-Leibler (KL) divergence [16] is used to measure the histogram distance:

$$
\begin{aligned}
D & =D_{K L}\left(H_{t} \| \hat{H}_{t}\right)+D_{K L}\left(\hat{H}_{t} \| H_{t}\right) \\
& =\sum_{i} \log \left(\frac{H_{t}(i)}{\hat{H}_{t}(i)}\right) H_{t}(i)+\sum_{i} \log \left(\frac{\hat{H}_{t}(i)}{H_{t}(i)}\right) \hat{H}_{t}(i)
\end{aligned}
$$

where $H_{t}$ is the histogram of the target image $I_{t}, \hat{H}_{t}$ is the histogram of the transformed image $\hat{I}_{t}$, and $i$ is the index of each histogram bins up to $2^{12}$ (equal to the maximal level of the raw image). This is computed per color channel with the mean reported as the KL divergence distance.

The comparison between Adobe DNG, Hong et al. [13], and our approach is given in Table 3 and Table 4 . We tested on six different cameras: Canon 1D Mark III, Nikon D40, Sony $\alpha 57$, Olympus E-PL6, Panasonic GX1, and Samsung NX2000. For each pair of cameras, eight pairs of raw images are examined and the mean values of the KL divergence distances are shown in Table 3 and Table 4 Our proposed method performs better than the other two methods. Additional results provided in the supplementary material.

\section{Example application}

Here we show an application of the raw-to-raw conversion using our approach to mosaic images captured from different cameras. Three different cameras Nikon D40, Sony $\alpha 57$, and Canon $1 \mathrm{D}$ are used. All three images are taken under the same exposure settings. Mosaicing without raw-to-raw conversion is shown in the top row of Figure 8 . The Canon and Nikon images are converted to be in the Sony rgb-raw colorspace based on our pre-computed
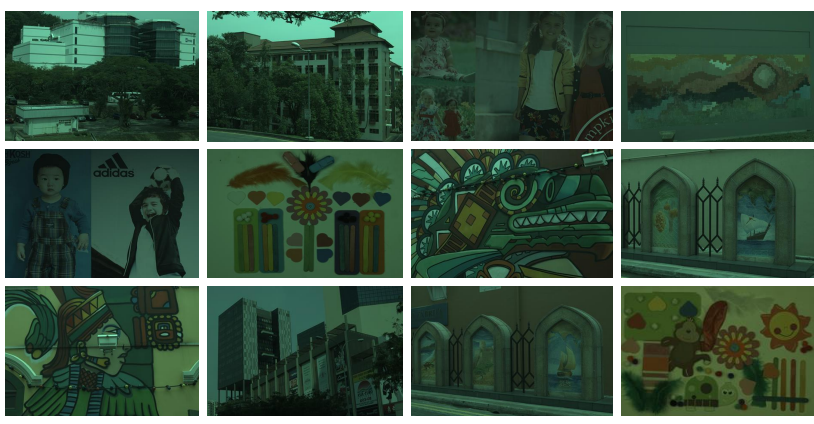

Figure 7. The figure shows example images of outdoor image set.
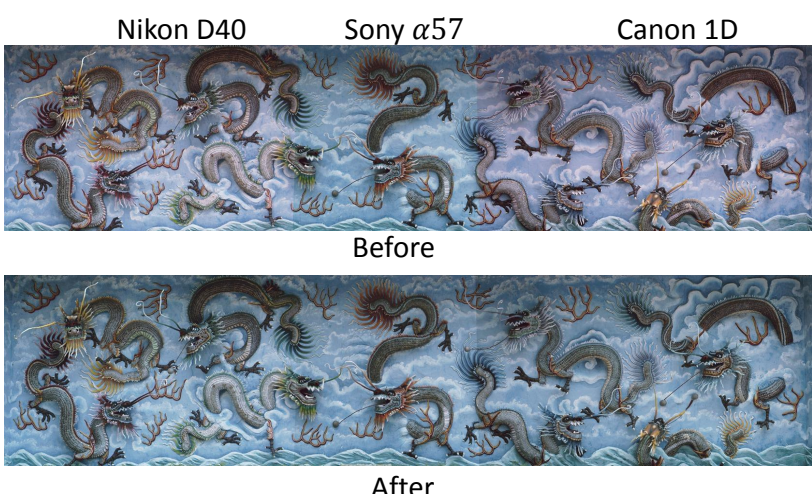

Figure 8. This figure shows an example of image mosaicing application. Three different cameras Nikon D40, Sony $\alpha 57$, and Canon $1 \mathrm{D}$ are used. This figure shows the comparison before and after conversion. All the images are converted to the raw-rgb space of the Sony $\alpha 57$. These mosaics have been adjusted by a gamma for better visualization.

transformation using the color charts described in Section 4 (no other blending or color conversion is applied). Figure 8 shows that after raw-to-raw conversion the mosaic has better color continuity and lesser apparent seams.

\section{Concluding remarks}

This paper has examined the problem of converting between camera's raw-rgb colorspaces. We examined five different mapping models applied in a global and illuminationspecific manner. Our results show that illumination-specific approach gives the best results, but at the disadvantage of maintaining multiple transformations and categorizing input images to scene illumination. To overcome this problem, we proposed an illumination-independent method that uses white-balancing to canonicalize the illumination. This method allows us to perform the raw-to-raw mapping using only two linear transformations. We described the calibration and our mapping procedure and showed its effectiveness on a range of inputs and under different illuminations. We have also prepared a data set useful in further exploration of the raw-to-raw conversion problem. 


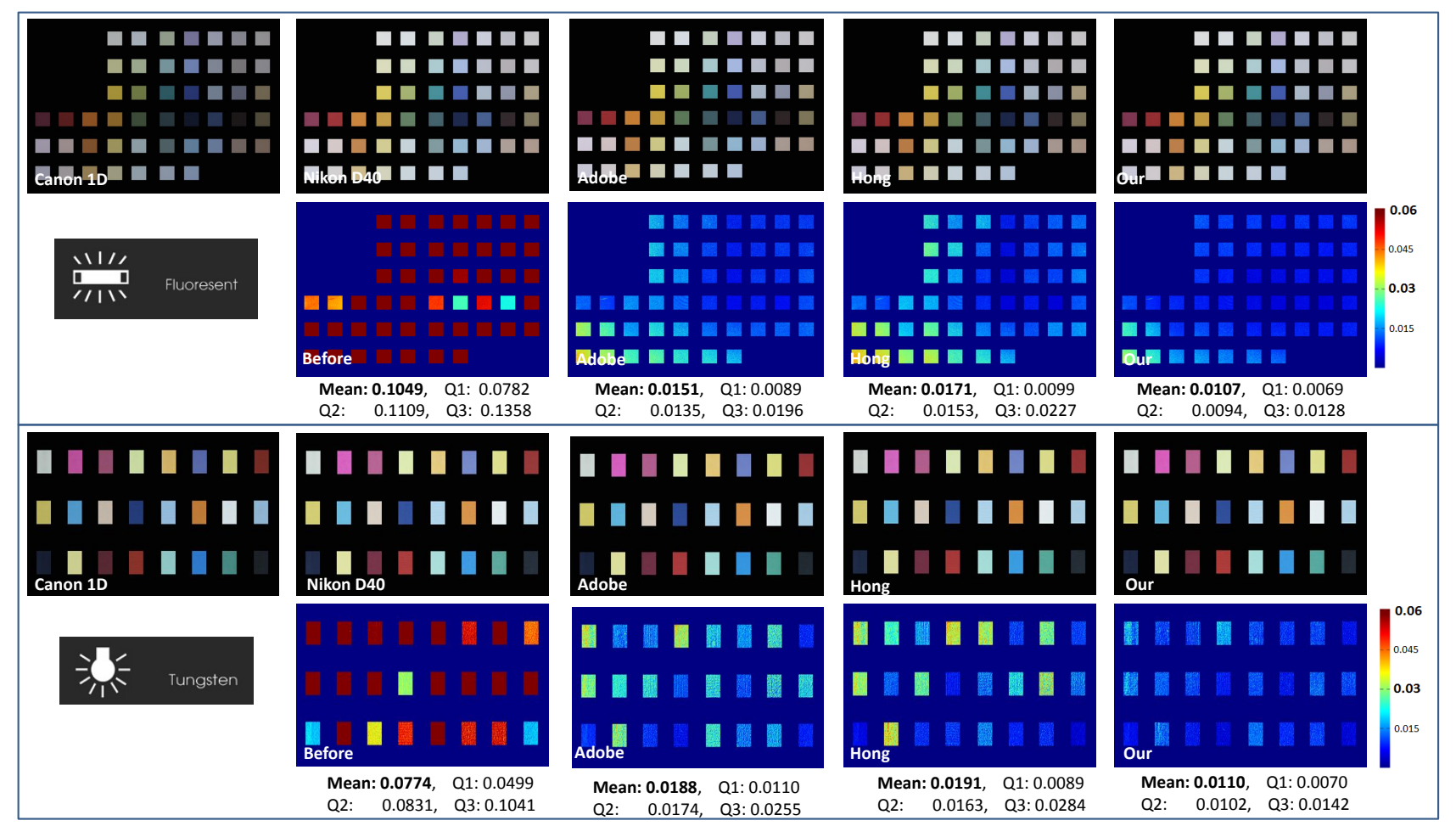

Figure 5. Comparison between all approaches. This shows the results on a Canon 1D and Nikon D40. Two lighting conditions are shown with the camera setting given to the DNG software. Results show the mean raw pixel errors (normalized) and the errors at the $25 \%$, $50 \%$ (median) and $75 \%$ quartiles (Q1, Q2, Q3).

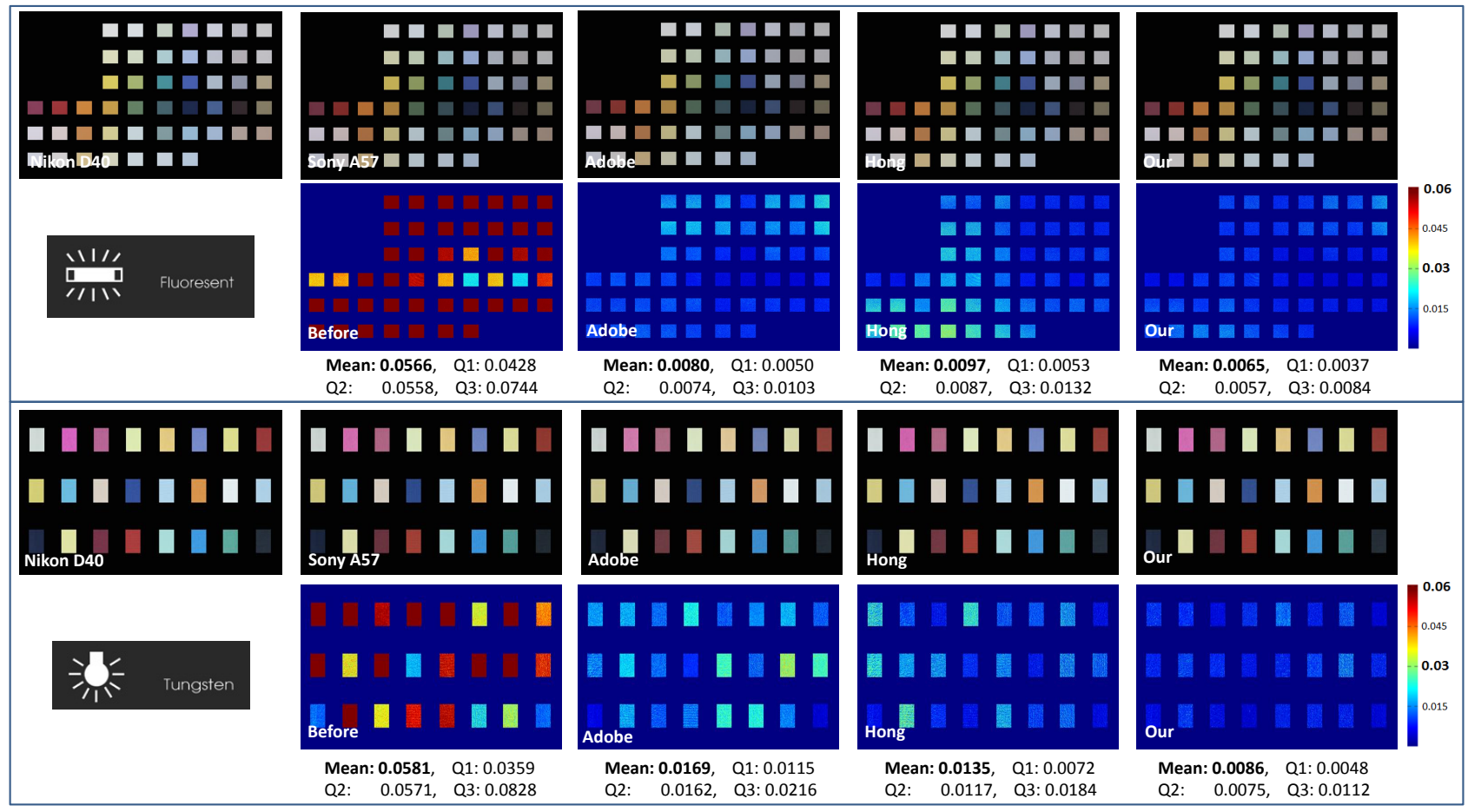

Figure 6. Comparison between all approaches. This shows the results on a Nikon D40 and a Sony $\alpha 57$. Two lighting conditions are shown with the camera setting given to the DNG software. Results show the mean raw pixel errors (normalized) and the errors at the $25 \%$, $50 \%$ (median) and 75\% quartiles (Q1, Q2, Q3). 


\begin{tabular}{|c|c|c|c|c|c|c|c|c|c|c|c|c|}
\hline & \multicolumn{4}{|c|}{ Canon1D } & \multicolumn{4}{|c|}{ Nikon D40 } & \multicolumn{4}{|c|}{ Sony $\alpha 57$} \\
\hline & Before & Adobe & Hong & Our & Before & Adobe & Hong & Our & Before & Adobe & Hong & Our \\
\hline Canon 1D & - & - & - & - & 0.8742 & 0.2251 & 0.0906 & 0.0543 & 0.4415 & 0.2793 & 0.1471 & 0.1451 \\
\hline Nikon D40 & 0.8742 & 0.1912 & 0.1091 & 0.0599 & - & - & - & - & 0.6901 & 0.1870 & 0.1983 & 0.1623 \\
\hline Sony $\alpha 57$ & 0.4415 & 0.1368 & 0.0527 & 0.0368 & 0.6901 & 0.0753 & 0.0802 & 0.0545 & - & - & - & - \\
\hline
\end{tabular}

Table 3. The table shows the comparisons of histogram distance computed by the equation 5 between all the approaches from three cameras: Canon 1D, Nikon D40, and Sony $\alpha 57$.

\begin{tabular}{|c|c|c|c|c|c|c|c|c|c|c|c|c|}
\hline & \multicolumn{4}{|c|}{ Olympus E-PL6 } & \multicolumn{4}{|c|}{ Panasonic GX1 } & \multicolumn{4}{|c|}{ Samsung NX2000 } \\
\hline & Before & Adobe & Hong & Our & Before & Adobe & Hong & Our & Before & Adobe & Hong & Our \\
\hline Olympus & - & - & - & - & 0.9450 & 0.1787 & 0.0750 & 0.0354 & 0.6661 & 0.2584 & 0.2630 & 0.0373 \\
\hline Panasonic & 0.9450 & 0.4529 & 0.2757 & 0.0162 & - & - & - & - & 0.3212 & 0.0951 & 0.2866 & 0.0475 \\
\hline Samsung & 0.6661 & 0.2278 & 0.1995 & 0.0236 & 0.3212 & 0.0667 & 0.0722 & 0.0583 & - & - & - & - \\
\hline
\end{tabular}

Table 4. The table shows the comparisons of histogram distance computed by the equation 5 between all the approaches from three cameras: Olympus E-PL6, Panasonic GX1, and Samsung NX2000.

\section{Acknowledgement}

This study was funded by A*STAR grant no. 1121202020 .

\section{References}

[1] Adobe. Digital negative (dng) specification. http://www.adobe.com/support/downloads/ dng/dng_sdk.html

[2] G. Buchsbaum. A spatial processor model for object colour perception. Journal of The Franklin Institute, 310(1):1-26, 1980.

[3] M. D. Buhmann. Radial basis functions: theory and implementations, volume 12. Cambridge University Press, 2003.

[4] A. Chakrabarti, K. Hirakawa, and T. Zickler. Color constancy with spatio-spectral statistics. IEEE Transactions on Pattern Analysis and Machine Intelligence, 34(8):15091519, 2012.

[5] A. Chakrabarti, D. Scharstein, and T. Zickler. An empirical camera model for internet color vision. In British Machine Vision Conference, 2009.

[6] D. Cheng, D. K. Prasad, and M. S. Brown. Illuminant estimation for color constancy: Why spatial domain methods work and the role of the color distribution. Journal of Optical Society America A, 31(5), 2014.

[7] H. Chong, S. Gortler, and T. Zickler. The von kries hypothesis and a basis for color constancy. In International Conference on Computer Vision, 2007.

[8] P. E. Debevec and J. Malik. Recovering high dynamic range radiance maps from photographs. In SIGGRAPH, pages 369-378, 1997.

[9] M. Diaz and P. Sturm. Radiometric calibration using photo collections. In International Conference on Computational Photography, 2011.

[10] G. D. Finlayson and G. Schaefer. Solving for colour constancy using a constrained dichromatic reflection model. International Journal of Computer Vision, 42(3):127-144, 2001.
[11] D. A. Forsyth. A novel algorithm for color constancy. International Journal of Computer Vision, 5(1):5-35, 1990.

[12] A. Gijsenij, T. Gevers, and J. van de Weijer. Computational color constancy: Survey and experiments. IEEE Transactions on Image Processing, 20(9), 2011.

[13] G. Hong, M. R. Luo, and P. A. Rhodes. A study of digital camera colorimetric characterization based on polynomial modeling. Color Res. \& Application, 26(1):76-84, 2001.

[14] J. Jiang, D. Liu, J. Gu, and S. Susstrunk. What is the space of spectral sensitivity functions for digital color cameras? In IEEE Workshop on Applications of Computer Vision (WACV), pages 168-179, 2013.

[15] S. J. Kim, H. T. Lin, Z. Lu, S. Susstrunk, S. Lin, and M. S. Brown. A new in-camera imaging model for color computer vision and its application. IEEE Transactions on Pattern Analysis and Machine Intelligence, 34(12):2289-2302, 2012.

[16] S. Kullback and R. A. Leibler. On information and sufficiency. The Annals of Mathematical Statistics, 22(1):79-86, 1951.

[17] F. Martinez-Verdu, J. Pujol, and P. Capilla. Characterization of a digital camera as an absolute tristimulus colorimeter. Journal of Imaging Science and Technology, 47(4):279-295, 2003.

[18] D. K. Prasad, R. Nguyen, and M. S. Brown. Quick approximation of cameras spectral response from casual lighting. In International Conference on Computer Vision Workshops (ICCVW 2013), 2013.

[19] C. E. Rasmussen. Gaussian processes for machine learning. 2006.

[20] P. Urban, M. Desch, K. Happel, and D. Spiehl. Recovering camera sensitivities using target-based reflectances captured under multiple LED-illuminations. In Workshop on Color Image Processing, pages 9-16, 2010.

[21] Y. Xiong, K. Saenko, T. Darrell, and T. Zickler. From pixels to physics: Probabilistic color de-rendering. In International Conference on Computer Vision and Pattern Recognition, 2012. 